Mathematical Research Letters 5, 137-148 (1998)

\title{
OSCILLATION AND BOUNDARY CURVATURE OF HOLOMORPHIC CURVES IN $\mathbb{C}^{n}$
}

\author{
D. NADleR AND S. YAKOVEnKo
}

\begin{abstract}
The number of isolated intersections between a smooth curve in Euclidean space and an arbitrary hyperplane can be majorized by a weighted sum of integral Frenet curvatures of the curve. In the complex Hermitian space one can derive a similar result for holomorphic curves but with much better weights.

The proof of this result is based on a generalization of the Milnor-Fáry theorem for complex Hermitian spaces: the expected integral curvature of a random hyperplanar Hermitian orthogonal projection of a smooth curve in $\mathbb{C}^{n}$ is equal to the integral curvature of the projected curve itself.

In the appendix we show how this technique allows one to improve the known estimates for real analytic curves in Euclidean space.
\end{abstract}

\section{Introduction}

A smooth real curve with bounded integral Frenet curvatures may have only a limited number of isolated intersections with an affine hyperplane: the upper bound is given by a certain weighted sum of the integral curvatures and the number of generalized inflections, the points of the curve at which the osculating frame degenerates [1]. The purpose of this short note is to establish an analog of this result for holomorphic curves in the complex space $\mathbb{C}^{n}$ equipped with the standard Hermitian structure.

Let $U \Subset \mathbb{C}$ be a simply connected domain bounded by a smooth curve $\gamma=\partial U$. Let $F: \bar{U} \rightarrow \mathbb{C}^{n}$ be a holomorphic curve and denote by $\Gamma: \gamma \rightarrow \mathbb{C}^{n}$ the restriction of $F$ to $\gamma$; this smooth curve in the complex space is the boundary of $F$. (When the parameterization does not play a role, we will also use $\Gamma$ to refer to the image of $\gamma$ under $F$ ). Let $\boldsymbol{\Omega}(F)$ be the maximal number of isolated intersections between the curve $F$ and a complex affine hyperplane in $\mathbb{C}^{n}$. Obviously, in all nontrivial cases $\boldsymbol{\Omega}(F) \geqslant n$, since the plane can pass through any $n$ points on $F$.

Our main result is an upper bound for $\boldsymbol{\Omega}(F)$ in terms of a weighted sum of the integral curvature $K(\gamma)$ of the boundary $\gamma=\partial U$, and the integral complex Frenet curvatures $\mathbf{K}_{j}(\Gamma)$ of the boundary $\Gamma=\partial F$ (the precise definition of these curvatures is given in $\S 2.1)$.

Received May 10, 1997. 
Theorem 1. The number of isolated intersections between a holomorphic curve $F: U \rightarrow \mathbb{C}^{n}$ and any affine complex hyperplane, does not exceed the weighted sum of integral complex Frenet curvatures of the boundary $\Gamma=\partial F$ and $\gamma=\partial U$ :

$$
2 \pi \boldsymbol{\Omega}(F) \leqslant 2 n K(\gamma)+4 \mathbf{K}_{1}(\Gamma)+\cdots+4 \mathbf{K}_{n-2}(\Gamma)+3 \mathbf{K}_{n-1}(\Gamma)+\mathbf{K}_{n}(\Gamma) .
$$

Remark. Except for the first term $2 n K(\gamma)$ in the above sum, the sequence of weights appearing in the inequality is bounded uniformly over the dimension $n$ of the ambient space. For smooth curves in Euclidean space the weights in [1] were growing as $O(n)$.

In fact, the tools developed in this paper allow one to improve rather substantially the upper bound for the number of intersections between a real analytic curve and an affine hyperplane in Euclidean space. The formulation of this result appears in the Appendix.

\section{Differential and integral geometry of smooth curves in the complex space}

2.1. Frenet formulas for smooth curves in the Hermitian space. The Frenet formulas for smooth curves in the complex Hermitian space are derived in the same way as their real counterparts. Let $\Gamma:[0, \ell] \rightarrow \mathbb{C}^{n}, t \mapsto x(t)=$ $\left(x_{1}(t), \ldots, x_{n}(t)\right)$ be a sufficiently smooth curve, parameterized by the arclength. Denote by $v_{j}:[0, \ell] \rightarrow \mathbb{C}^{n}$ its $j$ th derivative, $v_{j}=d^{j} x / d t^{j}$, for $j=1, \ldots, n$. In the generic case the vectors $v_{1}, \ldots, v_{n}$ are linearly independent at every point of $\Gamma$, and span the osculating flag $E_{1} \subset E_{2} \subset \cdots \subset E_{n-1} \subset E_{n}=\mathbb{C}^{n}$, where $E_{k}=\mathbb{C} v_{1}+\cdots+\mathbb{C} v_{k}$ (all subspaces of the flag depend on $t$, but we usually omit this dependence in the notations).

In general, there is no canonical choice of a Hermitian orthogonal frame $\left\{e_{1}(t)\right.$, $\left.\ldots, e_{n}(t)\right\}$ spanning the same flag (so that $E_{k}=\mathbb{C} e_{1}+\cdots+\mathbb{C} e_{k}$ ). However, under the additional assumption that $v_{k}=\beta_{k} e_{k} \bmod E_{k-1}$, with $\beta_{k}>0$, for all $k=1, \ldots, n$, the choice of the Frenet frame $\left\{e_{1}, \ldots, e_{n}\right\}$ is unique at any time $t$. In what follows, we will often denote the Frenet frame by $\left\{e_{1}, \ldots, e_{n}\right\}$ without further comment.

In the same way as for curves in the real space, the derivative $\dot{e}_{k}(t)$ will belong to $E_{k+1}$. Since the frame is Hermitian orthogonal, we can write

$$
\dot{e}_{k}=-\varkappa_{k-1} e_{k-1}+i \lambda_{k} e_{k}+\varkappa_{k} e_{k+1}
$$

with real $\lambda_{1}=\lambda_{1}(t), \ldots, \lambda_{n}=\lambda_{n}(t)$ and positive $\varkappa_{1}=\varkappa_{1}(t), \ldots, \varkappa_{n-1}=$ $\varkappa_{n-1}(t)$ (for $k=1$ and $k=n$ there will be only two terms in the right hand side of (1)). These relations constitute the Frenet formulas for smooth curves in the complex space, and coincide with the classical Frenet formulas if the curve belongs to the real subspace $\mathbb{R}^{n} \subset \mathbb{C}^{n}$. In this special case, all $\lambda_{k}$ are zero, and 
$\varkappa_{k}$ turn into the Frenet curvatures. In general, to restore the original velocities $v_{k}$ from the Frenet frame, one may use the identities

$$
\begin{aligned}
& v_{k}=\beta_{k} e_{k}+B_{k} e_{k-1} \bmod E_{k-2}, \quad k=1, \ldots, n, \\
& v_{n+1}:=\dot{v}_{n}=B_{n+1} e_{n} \bmod E_{n-1},
\end{aligned}
$$

where the coefficients $\beta_{k} \in \mathbb{R}_{+}, k=1, \ldots, n$, and $B_{k} \in \mathbb{C}, k=2, \ldots, n+1$, are defined by the identities

$$
\left(\beta_{k+1} / \beta_{k}\right)=\varkappa_{k}, \quad \operatorname{Im}\left(B_{k+1} / \beta_{k}\right)=\Lambda_{k}:=\lambda_{1}+\cdots+\lambda_{k}
$$

Indeed, differentiating the expression for $v_{k}$ with yet undefined $\beta_{k}$ and $B_{k}$, by virtue of (1), we have $\beta_{k+1} e_{k+1}+B_{k+1} e_{k}=\beta_{k}\left(\varkappa_{k} e_{k+1}+i \lambda_{k} e_{k}\right)+\dot{\beta}_{k} e_{k}+$ $B_{k} \varkappa_{k-1} e_{k} \bmod E_{k-1}$. Thus $\beta_{k+1}=\varkappa_{k} \beta_{k}$ and $\operatorname{Im} B_{k+1}=\beta_{k} \lambda_{k}+\varkappa_{k-1} \operatorname{Im} B_{k-1}$, since $\beta_{k}$ is always real and hence $\operatorname{Im} \dot{\beta}_{k}=0$. These two identities in turn imply $\operatorname{Im} B_{k+1} / \beta_{k}=\operatorname{Im} B_{k} / \beta_{k-1}+\lambda_{k}$, and, taking into account the "initial conditions" $\beta_{1}=\left\|v_{1}\right\|=1, B_{1}=0$, we arrive at the formulas (3).

Definition. The integral complex Frenet curvatures of orders $k=1, \ldots, n$ of a smooth curve $\Gamma$ in the complex space $\mathbb{C}^{n}$ are the integrals against the arclength,

$$
\mathbf{K}_{k}(\Gamma)=\int_{\Gamma} \sqrt{\Lambda_{k}^{2}+\varkappa_{k}^{2}} d \ell, \quad k=1, \ldots, n-1 ; \quad \mathbf{K}_{n}(\Gamma)=\int_{\Gamma}\left|\Lambda_{n}\right| d \ell .
$$

Remarks. There are several instances in which the complex case differs from the real one.

1. The osculating frame for a curve in the real space typically degenerates at isolated points, whereas in the complex space the osculating frame is typically nondegenerate. Indeed, the determinant of the square matrix with the vectors $v_{1}, \ldots, v_{n}$ as columns, is a complex-valued smooth function of the real parameter $t$, and by a $C^{n}$-small perturbation of the curve one can destroy all zeros of this function on the segment $[0, \ell]$. Similar considerations in the real case prove only that the roots of the determinant are non-critical hence isolated (these roots correspond to the inflection points as defined in [1]).

2. As mentioned above, the formulas for the complex curvatures $\mathbf{K}_{j}(\Gamma)$, $j=1, \ldots, n-1$, coincide with their real counterparts for curves lying in the real subspace. The expression for $\mathbf{K}_{n}$ is a complex analog (and a majorant) for the number of generalized inflection points [3] in the case when $\Gamma=\partial F$ is the boundary of a holomorphic curve $F$.

3. E. Calabi in [2] derived the Frenet theory for holomorphic curves in the Hermitian space. For a holomorphic non-hyperplanar curve $F: U \rightarrow \mathbb{C}^{n}$ one can define $n-1$ real analytic functions $\rho_{k}: U \rightarrow \mathbb{R}$ by the same formulas as the real Frenet curvatures. It turns out that the first of them, $\rho_{1}(z)$, is essentially the Gaussian curvature of the induced metric. The other (higher) curvatures 
are uniquely defined by $\rho_{1}$ and the embedding of the curve into $\mathbb{C}^{n}$ is uniquely determined by $\rho_{1}$ (hence by the intrinsic geometry) modulo a Hermitian rotation.

One can easily show that if a smooth curve $\Gamma$ lies on a holomorphic curve $F$, then $\left|\lambda_{1}\right|$ is the geodesic curvature of $\Gamma$ in the induced Riemannian metric on $F$ (for example, if $F$ is flat, then $\Gamma$ may be treated as a planar curve, and $\mathbf{K}_{1}=K(\Gamma)$ ). In addition, for geodesic curves the first curvature $\mathbf{K}_{1}$ coincides with the integral Calabi curvature $\int_{0}^{\ell}\left|\rho_{1}(t)\right| d t$. The relations between the higher Calabi curvatures of a holomorphic curve and the complex Frenet curvatures of smooth arcs on it are not clear.

2.2. Variation of argument, Voorhoeve index, pseudoinflection and their properties. In this subsection we introduce several definitions all closely related to the variation of argument of a complex-valued function.

2.2.1. Variation of argument along a planar curve. If $\gamma \subset \mathbb{C} \backslash\{0\}$, given by $t \mapsto x(t)=r(t) \exp i \varphi(t)$, is a continuous curve on the complex plane, avoiding the origin, then the total variation of its argument $\mathbf{V}(\gamma)$ is well defined. For a smooth curve it is equal to the integral

$$
\int_{0}^{\ell}|d \varphi(t) / d t| d t=\int_{0}^{\ell}\left|\frac{d}{d t} \operatorname{Arg} x(t)\right| d t=\int_{0}^{\ell}\left|\operatorname{Im}\left(x^{-1}(t) \cdot \dot{x}(t)\right)\right| d t,
$$

which majorizes the (scaled by $2 \pi$ ) winding number of the curve around the origin.

2.2.2. Voorhoeve index of an analytic function. Let $U \Subset \mathbb{C}$ be a domain bounded by a smooth curve $\gamma=\partial U$, and $f: U \mapsto \mathbb{C}$ a complex-valued function such that $f$ is never zero on the boundary $\gamma$. The Voorhoeve index [3] of $f$ with respect to the curve $\gamma$ is defined to be the total variation of argument of $f$ along $\gamma$ : $\mathbf{V}_{\gamma}(f):=\mathbf{V}(f(\gamma))$. In other words, it is equal to the above introduced variation of argument of the image curve $f(\gamma)$. Obviously, $\mathbf{V}_{\gamma}(c f)=\mathbf{V}_{\gamma}(f)$ for any $0 \neq c \in \mathbb{C}$ (scaling and rotational invariance).

Example 1. Let $z(t)$ be any parameterization of $\gamma$ so long as $\dot{z}(t)$ is never zero. Then unwinding the definitions, one obtains $\mathbf{V}_{\gamma}(\dot{z})=K(\gamma)$, the integral curvature of the boundary $\gamma$, occurring in the main theorem.

Of course, we will be most interested in the Voorhoeve index of meromorphic functions with no poles on $\gamma$. In the particular case when $f$ is analytic throughout $U$, we know by the argument principle that the number of zeros of $f$ in $U$ is equal to the winding number of $f(\gamma)$ around the origin. Since the Voorhoeve index $\mathbf{V}_{\gamma}(f)$ is a majorant for the winding number (in fact, $2 \pi$ times the winding number), it is in turn a majorant for the number of zeros of $f$.

Example 2. Suppose that $f$ is meromorphic on an open neighborhood of a real segment $[0, \ell]$ and takes real values on it (except for poles). Denote by $U_{\epsilon}$ the $\epsilon$-neighborhood of $[0, \ell]$ in $\mathbb{C}$, and let $\gamma_{\epsilon}=\partial U_{\epsilon}$. Then it is clear, see [4], that

$$
\lim _{\epsilon \rightarrow 0^{+}} \mathbf{V}_{\gamma_{\epsilon}}(f)=2 \pi(\#\{f=0\}+\#\{f=\infty\}) .
$$


For $f$ real analytic on $[0, \ell]$ the above limit is $2 \pi$ times the number of zeros of $f$ on the segment.

The reason for introducing the Voorhoeve index, as opposed to working with the winding number of $f(\gamma)$, is the following. Unlike the winding number of $f(\gamma)$, the closely related Voorhoeve index behaves much more nicely under derivation. The following properties, generalizing the Rolle lemma for real functions of one variable, were proved in [3].

Rolle-Voorhoeve lemma. If $f, g$ are holomorphic in a neighborhood of $\gamma$, then

$$
\begin{array}{ll}
\mathbf{V}_{\gamma}(f) \leqslant \mathbf{V}_{\gamma}\left(f^{\prime}\right)+K(\gamma) & \text { (the Rolle inequality), } \\
\left|\mathbf{V}_{\gamma}(f)-\mathbf{V}_{\gamma}(g)\right| \leqslant \mathbf{V}_{\gamma}(f g) \leqslant \mathbf{V}_{\gamma}(f)+\mathbf{V}_{\gamma}(g) & \text { (the triangle inequality), } \\
\mathbf{V}_{\gamma}(1 / f)=\mathbf{V}_{\gamma}(f) & \text { (symmetry). }
\end{array}
$$

where $K(\gamma)$ is the integral curvature of the boundary (for example, $2 \pi$ if $\gamma$ is convex).

Remark. In fact, the Rolle inequality above is a particular case of a more general inequality relating rotation and curvature of smooth curves, not necessarily closed ones [3]. Another particular case concerns periodic functions.

Let $f$ be meromorphic and real on the real axis as in Example 2 above. Assume in addition that $f$ is $\ell$-periodic, i.e. $f(t+\ell) \equiv f(t)$, and $f(0) \neq 0, \infty$. Denote by $\mathbf{N}(f)$ the sum of the number of zeros and poles on the segment $[0, \ell]$ (or any other segment of length $\ell$ with nonsingular endpoints). Then from the general inequality [3] it follows that $\mathbf{N}(f) \leqslant \mathbf{N}\left(f^{\prime}\right)$.

2.2.3. Pseudoinflection of a non-planar smooth curve in $\mathbb{C}^{n}$. Now let $\Gamma:[0, \ell] \rightarrow$ $\mathbb{C}^{n}$ be a sufficiently generic smooth curve. Then the osculating vectors $v_{1}, \ldots, v_{n}$ are independent everywhere and their wedge product $v_{1}(t) \wedge \cdots \wedge v_{n}(t)$ can be identified with a nonvanishing function $\Delta=\Delta_{\Gamma}:[0, \ell] \rightarrow \mathbb{C} \backslash\{0\}$ with complex values, well defined modulo a constant factor. If $x_{1}(t), \ldots, x_{n}(t)$ are the coordinate functions of the embedding $\Gamma$ in $\mathbb{C}^{n}$, then $\Delta$ is the Wronski determinant of the time derivatives $\dot{x}_{1}(t), \ldots, \dot{x}_{n}(t)$. If the curve were in Euclidean space, then zeros of $\Delta$ would correspond to (generalized) inflection points, as introduced in [1]. In the complex setting we replace the number of zeros by the variation of argument of $\Delta$ considered as a curve $[0, \ell] \rightarrow \mathbb{C} \backslash\{0\}$.

Definition. The pseudoinflection of a smooth non-hyperplanar curve $\Gamma$ in $\mathbb{C}^{n}$ is

$\mathbf{I}(\Gamma)=\mathbf{V}\left(\Delta_{\Gamma}\right)=\int_{0}^{\ell}\left|\frac{d}{d t} \operatorname{Arg}\left(v_{1} \wedge \cdots \wedge v_{n}\right)\right| d t=\int_{0}^{\ell}\left|\operatorname{Im} \frac{v_{1} \wedge \cdots \wedge v_{n-1} \wedge \dot{v}_{n}}{v_{1} \wedge \cdots \wedge v_{n-1} \wedge v_{n}}\right| d t$.

One can immediately see that this definition does not depend on the choice of parameterization: any change of parameter $t \mapsto \tau=\tau(t)$ with $d \tau / d t>0$ results 
in $\Delta(t)$ being replaced by $r(\tau) \cdot \Delta(t(\tau))$, with $r(\tau)$ a real-valued positive function, hence the variation of argument remains the same. Moreover, the number $\mathbf{I}(\Gamma)$ is invariant by Hermitian orthogonal rotations.

2.2.4. Pseudoinflection of $k$-planar curves. The above definition makes sense on the assumption that the curve $\Gamma$ is not hyperplanar, more precisely, that the wedge product $v_{1}(t) \wedge \cdots \wedge v_{n}(t)$ is nonvanishing on $[0, \ell]$.

However, below we will have to consider the opposite case and compute the pseudoinflection of $k$-planar curves, i.e. curves by construction belonging to some $k$-plane $L \subset \mathbb{C}^{n}$ with $k<n$. Formally this case can be incorporated into the above definition by identifying $L$ with a copy of $\mathbb{C}^{k}$, e.g. with the complex $k$ space spanned by the first $k$ coordinate axes. In this case $\Delta_{\Gamma}$ can be identified with the Wronski determinant of the time derivatives of the first $k$ coordinate functions $\dot{x}_{1}(t), \ldots, \dot{x}_{k}(t)$.

Alternatively one may choose any $n-k$ vectors $p_{k+1}, \ldots, p_{n}$ in $\mathbb{C}^{n}$ spanning a subspace transversal (complementary) to $L$. Then the wedge product $v_{1} \wedge \cdots \wedge$ $v_{k} \wedge p_{k+1} \wedge \cdots \wedge p_{n}$ will be well-defined modulo a constant factor depending on the choice of $\left\{p_{k+1}, \ldots, p_{n}\right\}$. The variation of argument of this extended wedge product will be a rotational invariant of $\Gamma$, independent of the parameterization, and obviously coinciding with the variation of argument of $\Delta_{\Gamma}$ as introduced above.

Note that for any real analytic curve $\Gamma$ not reducible to one point, there always exists the dimension $k \leqslant n$ such that $\Gamma$ is $k$-planar, i.e. $\Gamma \subset L, \operatorname{dim} L=k$, and the $k$-pseudoinflection of $\Gamma$ (relative to $L$ ) is well-defined and finite. Sometimes we will write $\mathbf{I}(\Gamma ; L)$ to stress the fact that $\Gamma \subset L$.

2.2.5. Real and complex Wronskians. Let $F: U \rightarrow \mathbb{C}^{n}, z \mapsto\left(x_{1}(z), \ldots, x_{n}(z)\right)$ be a holomorphic curve, $\gamma=\partial U, \Gamma=F(\gamma)$. Denote by $W=W_{F}(z)$ the Wronski determinant of the $n$ derivatives $x_{1}^{\prime}(z), \ldots, x_{n}^{\prime}(z)$; together with these derivatives, $W$ is holomorphic in $U$. There is an obvious relationship between $W(z)$ and the function $\Delta_{\Gamma}(z)$ as defined in $\S 2.2 .3$ : for an arbitrary parameterization $[0, \ell] \rightarrow \mathbb{C}$, $t \mapsto z(t)$, of the boundary curve $\gamma$, the chain rule yields

$$
\Delta_{\Gamma}(t)=W_{F}(z(t)) \cdot[\dot{z}(t)]^{1+2+\cdots+n} .
$$

2.3. Integral geometry and geometric probability. Let $G_{k}(n)$ be the Grassman manifold of complex $k$-planes in $\mathbb{C}^{n}$. It is a complex compact manifold with transitive $S U(n)$-action, and there is a unique normalized $S U(n)$ invariant measure in $G_{k}(n)$ that will be usually denoted by $d \mu_{k, n}$ or simply $d \mu$. In the particular case $n=2, k=1$ we have the Fubini-Studi measure on the projective line $\mathbb{C} P^{1}=G_{1}(2)$, that in the affine coordinate $z$ takes the form $d \mu(z)=\frac{1}{\pi}\left(1+|z|^{2}\right)^{-2} d z \wedge d \bar{z}$.

We begin with several simple statements concerning behavior of the canonical measures under geometric constructions. Each subspace $E \subset \mathbb{C}^{n}$ of dimension $d$, identified with $\mathbb{C}^{d}$, defines two "maps" from $G_{k}(n)$ to $G_{k-n+d}(d)$ and to $G_{k}(d)$ respectively, called intersection and projection: for almost any $k$-plane 
$L \in G_{k}(n)$ the intersection $\iota(L)=L \cap E$ is a subspace of dimension $k-n+d$ in $E \simeq \mathbb{C}^{d}$ and the Hermitian orthogonal projection $\pi(L)$ of $L$ onto $E$ parallel to $E^{\perp}$ is a $k$-dimensional subspace of $E$ (the nontransversal or badly projecting planes constitute a zero measure set of $G_{k}(n)$ and will be neglected in the sequel). This construction allows one to push forward the canonical measure from $G_{k}(n)$ to $G_{k-n+d}(d)$ and $G_{k}(d)$ respectively. Not surprisingly, these transferred measures coincide with the canonical measures on the respective Grassmanians. In terms of geometric probability, this becomes a claim on the uniformity of distributions of certain random $k$-planes.

Proposition $\mathbf{G}_{1}$. The intersection $\iota(L) \in G_{k-n+d}(d)$ and projection $\pi(L) \in$ $G_{k}(d)$ of a random uniformly distributed $k$-plane $L \in G_{k}(n)$ are uniformly distributed over the corresponding Grassmanians.

Any $(n-1)$-dimensional hyperplane $\Pi \in G_{n-1}(n)$ in the Hermitian space $\mathbb{C}^{n}$ naturally inherits the metric and $S U(n-1)$-invariant measure (and hence one can choose a random $k$-plane inside $\Pi$ ). The following is a claim concerning the conditional distribution.

Proposition $\mathbf{G}_{2}$. A random $k$-plane $L$, uniformly distributed inside a random uniformly distributed hyperplane $\Pi \in G_{n-1}(n)$, is also uniformly distributed in $G_{k}(n)$.

Proof. Both claims follow from the uniqueness of the $S U$-invariant measures on the Grassmanians.

\section{Average pseudoinflection and the Milnor-Fáry lemma in $\mathbb{C}^{n}$}

3.1. Average pseudoinflection of random projections. Let $\Gamma$ be a smooth curve in $\mathbb{C}^{n}$. For almost any $k$-plane $L \in G_{k}(n)$ the Hermitian orthogonal projection $\pi_{L}(\Gamma)$ of $\Gamma$ on $L$ parallel to $L^{\perp}$ will be a nonsingular $k$-planar curve, whose pseudoinflection will be denoted by $\mathbf{I}\left(\pi_{L}(\Gamma) ; L\right)$. After averaging over all $k$-planes (with respect to the canonical measure) this gives the quantity provisionally denoted by $I_{k}(\Gamma)$ :

$$
I_{k}(\Gamma)=\int_{G_{k}(n)} \mathbf{I}\left(\pi_{L}(\Gamma) ; L\right) d \mu(L) .
$$

Lemma 1. The pseudoinflection averaged over all $k$-dimensional Hermitian orthogonal projections, is equal to the $k$ th integral complex Frenet curvature:

$$
I_{k}(\Gamma)=\mathbf{K}_{k}(\Gamma) \quad \text { for all } k=1, \ldots, n
$$

Proof. Assuming the curve $\Gamma$ sufficiently smooth, denote by $\Delta_{L}(t)$ the wedge product (relative to the $k$-plane $L$ ) of the osculating vectors of the curve $\pi_{L}(\Gamma)$, as introduced in $\S 2.2 .4$. Then $\mathbf{I}\left(\pi_{L}(\Gamma) ; L\right)=\int_{\Gamma}\left|\operatorname{Im}\left(\Delta_{L}^{-1} \cdot \frac{d}{d t} \Delta_{L}\right)\right| d t$. 
1. Choose any frame $\left\{p_{k+1}, \ldots, p_{n}\right\}$ in $L^{\perp}$ (the Hermitian orthogonal subspace) so that, as usual modulo a scalar factor, $\Delta_{L}=v_{1} \wedge \cdots \wedge v_{k} \wedge p_{k+1} \wedge \cdots \wedge p_{n}$. Differentiating with respect to $t$, we observe that $\frac{d}{d t} \Delta_{L}=v_{1} \wedge \cdots \wedge v_{k-1} \wedge v_{k+1} \wedge$ $p_{k+1} \wedge \cdots \wedge p_{n}$. Substituting this into the definition of $I_{k}$ and changing the order of integration, we arrive to the identity

$$
I_{k}=\int_{0}^{\ell} d t \int_{G_{k}(n)}\left|\operatorname{Im} \frac{v_{1} \wedge \cdots \wedge v_{k-1} \wedge v_{k+1} \wedge p_{k+1} \wedge \cdots \wedge p_{n}}{v_{1} \wedge \cdots \wedge v_{k-1} \wedge v_{k} \wedge p_{k+1} \wedge \cdots \wedge p_{n}}\right| d \mu(L) .
$$

Note that for fixed $t$ the integrand is independent of the choice of the frame $\left\{p_{k+1}, \ldots, p_{n}\right\}$ in $L^{\perp}$, being a function of the relative position of the variable subspace $L$, the fixed subspace $E_{k-1}$ spanned by $\left\{v_{1}, \ldots, v_{k-1}\right\}$, and the pair of vectors $v_{k}, v_{k+1}$. Denote this function by $Z=Z\left(E_{k-1}, v_{k}, v_{k+1}, L\right)$ so that the integral takes the form $I_{k}=\int_{0}^{\ell} \int_{G_{k}(n)}\left|\operatorname{Im} Z\left(E_{k-1}, v_{k}, v_{k+1}, L\right)\right| d \mu(L)$.

2. By the above remarks, one can replace the collection $\left\{v_{1}, \ldots, v_{k-1}\right\}$ in (6) by any other frame spanning the same subspace $E_{k-1}$, in particular, by the Frenet frame $\left\{e_{1}, \ldots, e_{k-1}\right\}$. Using the freedom to choose the frame $\left\{p_{k+1}, \ldots\right.$, $\left.p_{n}\right\}$, we choose $p_{k+1}$, the first vector of this frame, to be in the intersection $L^{\perp} \cap E_{k+1}$. Now using (2) to express the vectors $v_{k}$ and $v_{k+1}$, we observe that the value of the integrand only depends upon the projection of $p_{k+1}$ onto the two-dimensional span $\mathbb{C} e_{k} \oplus \mathbb{C} e_{k+1} \subset E_{k+1}$, not upon $p_{k+1}$ itself. We denote this projection by $\zeta_{1} e_{k}+\zeta_{2} e_{k+1}$ so that $p_{k+1}=\zeta_{1} e_{k}+\zeta_{2} e_{k+1} \bmod E_{k-1}$. Furthermore, for the sake of readability, we set $\mathfrak{E}_{k-1}=e_{1} \wedge \cdots \wedge e_{k-1} \in \wedge^{k-1}\left(\mathbb{C}^{n}\right)$ and $\mathfrak{P}_{k+2}=p_{k+2} \wedge \cdots \wedge p_{n} \in \bigwedge^{n-k-1}\left(\mathbb{C}^{n}\right)$. With the new notation, we have

$$
\begin{aligned}
|\operatorname{Im} Z| & =\left|\operatorname{Im} \frac{\mathfrak{E}_{k-1} \wedge v_{k+1} \wedge p_{k+1} \wedge \mathfrak{P}_{k+2}}{\mathfrak{E}_{k-1} \wedge v_{k} \wedge p_{k+1} \wedge \mathfrak{P}_{k+2}}\right| \\
& =\left|\operatorname{Im} \frac{\mathfrak{E}_{k-1} \wedge\left(\beta_{k+1} e_{k+1}+B_{k+1} e_{k}\right) \wedge\left(\zeta_{1} e_{k}+\zeta_{2} e_{k+1}\right) \wedge \mathfrak{P}_{k+2}}{\mathfrak{E}_{k-1} \wedge\left(\beta_{k} e_{k}\right) \wedge\left(\zeta_{1} e_{k}+\zeta_{2} e_{k+1}\right) \wedge \mathfrak{P}_{k+2}}\right| \\
& =\left|\operatorname{Im}\left(\frac{B_{k+1}}{\beta_{k}}-\frac{\beta_{k+1}}{\beta_{k}} \cdot \frac{\zeta_{1}}{\zeta_{2}}\right)\right|=\left|\Lambda_{k}-\varkappa_{k} \operatorname{Im}\left(\zeta_{1} / \zeta_{2}\right)\right| .
\end{aligned}
$$

3. By virtue of Proposition $\mathrm{G}_{1}$, since $\mathbb{C} p_{k+1}=L^{\perp} \cap E_{k+1}$ is uniformly distributed in $E_{k+1}$, its projection onto the two-dimensional span $\mathbb{C} e_{k} \oplus \mathbb{C} e_{k+1} \subset$ $E_{k+1}$ is as well. Thus instead of averaging over the Grassmanian $G_{k}(n)$ in (6), we may compute the average of the function $|\operatorname{Im} Z|$ over all projections of $\mathbb{C} p_{k+1}$ on $\mathbb{C}^{2} \simeq \mathbb{C} e_{k} \oplus \mathbb{C} e_{k+1}$, in other words, over the projective plane $\mathbb{C} P^{1}$ equipped with the homogeneous coordinates $\zeta=\left[\zeta_{1}: \zeta_{2}\right] \in \mathbb{C} P^{1}$ and the canonical rotationally invariant measure. Denoting by $z=x+i y=\zeta_{1} / \zeta_{2} \in \mathbb{C}$ the affine chart and using the explicit form for the Fubini-Studi measure, we arrive to the explicit 
answer:

$$
\begin{aligned}
I_{k} & =\int_{0}^{\ell} d t \int_{\mathbb{C}}\left|\Lambda_{k}-\varkappa_{k} \operatorname{Im} z\right| \frac{d z \wedge d \bar{z}}{\pi\left(1+|z|^{2}\right)^{2}} \\
& =\int_{0}^{\ell} d t \int_{-\infty}^{+\infty}\left|\Lambda_{k}-\varkappa_{k} y\right| d y \int_{-\infty}^{+\infty} \frac{d x}{\pi\left(1+x^{2}+y^{2}\right)^{2}} \\
& =\int_{0}^{\ell} d t \int_{-\infty}^{+\infty} \frac{\left|\Lambda_{k}-\varkappa_{k} y\right|}{2\left(1+y^{2}\right)^{3 / 2}} d y=\int_{0}^{\ell} d t \sqrt{\Lambda_{k}^{2}+\varkappa_{k}^{2}}=\mathbf{K}_{k} .
\end{aligned}
$$

4. The case $k=n$ is to be treated separately, but in this case the averaging step disappears, and for the pseudoinflection of the curve we have by (2)

$$
I_{n}=\mathbf{I}=\int_{0}^{\ell}\left|\operatorname{Im} \frac{v_{1} \wedge \cdots \wedge v_{n-1} \wedge \dot{v}_{n}}{v_{1} \wedge \cdots \wedge v_{n-1} \wedge v_{n}}\right| d \ell=\int_{0}^{\ell}\left|\operatorname{Im} \frac{B_{n+1}}{\beta_{n}}\right| d \ell=\int_{\Gamma}\left|\Lambda_{n}\right| d \ell=\mathbf{K}_{n} .
$$

3.2. Average curvatures of hyperplanar projections. If $\Gamma$ is a real curve in $\mathbb{C}^{n}$ with integral curvatures $\mathbf{K}_{1}(\Gamma), \ldots, \mathbf{K}_{n}(\Gamma)$, then its Hermitian orthogonal projection onto a hyperplane $L \in G_{n-1}(n)$ has only $n-1$ curvatures $\mathbf{K}_{k}\left(\pi_{L}(\Gamma)\right)$, $k=1, \ldots, n-1$. The following result is the generalization of the Milnor-Fáry lemma on average curvatures [1, Lemma 1] for the Hermitian space.

Corollary 1 (Milnor-Fáry lemma for smooth curves in the Hermitian space). The expected curvature of a random Hermitian projection of a smooth curve is equal to the curvature of the initial curve:

$$
\mathbf{K}_{k}(\Gamma)=\int_{G_{n-1}(n)} \mathbf{K}_{k}\left(\pi_{L}(\Gamma)\right) d \mu(L) \quad \forall k=1, \ldots, n-1
$$

Proof. By Proposition $\mathrm{G}_{2}$, the same result will be obtained if instead of averaging over all $k$-planes from $G_{k}(n)$, one first chooses a hyperplane $L \in G_{n-1}(n)$, averages over all $k$-planes inside $L$ and then averages the result over all such hyperplanes.

Remark. The assertion of Corollary 1 cannot be reduced to the usual MilnorFáry lemma in $\mathbb{R}^{n}$ as in [1] by forgetting the complex structure. Even if one identifies $\mathbb{C}^{n}$ with $\mathbb{R}^{2 n}$ and reduces the complex Frenet curvatures to their real counterparts, the average in the above Corollary will be extended over only a small portion of all codimension-two real subspaces, namely, those coming from complex hyperplanes. 
Corollary 2. Let $\Gamma$ be a smooth curve in $\mathbb{C}^{n}, w_{1}>0, \ldots, w_{n}>0$ an arbitrary collection of weights. Then one can find a complete flag $E_{1} \subset \cdots \subset E_{n-1} \subset$ $E_{n}=\mathbb{C}^{n}$ of subspaces in such a way that $\sum_{k=1}^{n} w_{k} \mathbf{I}\left(\Gamma_{k}\right) \leqslant \sum_{k=1}^{n} w_{k} \mathbf{K}_{k}(\Gamma)$, where $\Gamma_{k}$ is the Hermitian orthogonal projection of $\Gamma$ on $E_{k}$, and $\mathbf{I}\left(\Gamma_{k}\right)=$ $\mathbf{I}\left(\Gamma_{k} ; E_{k}\right)$ the corresponding (relative) pseudoinflection.

Proof. The proof is essentially the same as in the real case [1], and goes by induction on the ambient dimension $n$. For $n=1$ the assertion is obvious, since the only existing curvature $\mathbf{K}_{1}$ coincides with the pseudoinflection $\mathbf{I}$. Suppose that for $n-1$ the claim is already justified, and consider the weighted sum $\mathbf{K}_{w}=$ $w_{n} \mathbf{K}_{n}+w_{n-1} \mathbf{K}_{n-1}+\cdots+w_{1} \mathbf{K}_{1}=w_{n} \mathbf{K}_{n}+\widehat{\mathbf{K}}_{w}$. The topmost curvature $\mathbf{K}_{n}$ coincides with the pseudoinflection $\mathbf{I}=\mathbf{I}\left(\cdot ; E_{n}\right)$, so consider the remaining part $\widehat{\mathbf{K}}_{w}=w_{n-1} \mathbf{K}_{n-1}+\cdots+w_{1} \mathbf{K}_{1}$ as a functional on smooth curves. Its average over all $(n-1)$-dimensional projections of the curve $\Gamma$ is equal to the value of $\widehat{\mathbf{K}}_{w}$ on $\Gamma$ by Corollary 1, and therefore by the mean value theorem there exists an $(n-1)$-plane $E_{n-1} \subset E_{n} \simeq \mathbb{C}^{n}$ such that $\widehat{\mathbf{K}}_{w}\left(\Gamma_{n-1}\right) \leqslant \widehat{\mathbf{K}}_{w}(\Gamma)$, where $\Gamma_{n-1}$ is the Hermitian orthogonal projection of $\Gamma$ on $E_{n-1}$. By the induction assumption, the flag $E_{1} \subset \cdots \subset E_{n-2}$ inside $E_{n-1}$ can be constructed as required so that $\widehat{\mathbf{K}}_{w}\left(\Gamma_{n-1}\right) \geqslant \sum_{j=1}^{n-1} w_{j} \mathbf{I}\left(\Gamma_{j} ; E_{j}\right)$, and this flag (appended by the space $E_{n-1}$ ), serves also the space $\mathbb{C}^{n}$, since

$$
\mathbf{K}_{w}(\Gamma)=w_{n} \mathbf{I}(\Gamma)+\widehat{\mathbf{K}}_{w}(\Gamma) \geqslant w_{n} \mathbf{I}(\Gamma)+\widehat{\mathbf{K}}_{w}\left(\Gamma_{n-1}\right) \geqslant w_{n} \mathbf{I}(\Gamma)+\sum_{j=1}^{n-1} w_{j} \mathbf{I}\left(\Gamma_{j}\right) .
$$

\section{Linear ordinary differential equations with analytic coefficients and the proof of the main theorem}

4.1. Complex zeros of linear combinations of holomorphic functions. Let $U \Subset \mathbb{C}$ be a simply connected domain bounded by a smooth curve $\gamma=$ $\partial U$ with integral curvature $K=K(\gamma)$, and $f_{1}, \ldots, f_{n}$ be an ordered $n$-tuple of functions analytic on the closure $\bar{U}=U \cup \gamma$. Denote by $W_{k}=W_{k}(z)$, $k=0,1, \ldots, n$, the Wronski determinant of the first $k$ functions: $W_{0} \equiv 1$, $W_{1}=f_{1}, W_{2}=f_{1} f_{2}^{\prime}-f_{2} f_{1}^{\prime}$ etc. It is known that any linear combination $f=\mu_{1} f_{1}+\cdots+\mu_{n} f_{n}$ satisfies the homogeneous linear ordinary differential equation of order $n$,

$$
\frac{W_{n}}{W_{n-1}} \cdot \partial \cdot \frac{W_{n-1}^{2}}{W_{n} W_{n-2}} \cdot \partial \cdots \partial \cdot \frac{W_{1}^{2}}{W_{2} W_{0}} \cdot \partial \cdot \frac{W_{0}}{W_{1}} \cdot f=0, \quad \partial=\frac{d}{d z},
$$

(the Frobenius formula), that can without loss of generality be reduced to the form containing only $n-1$ derivations,

$$
\frac{W_{n-1}^{2}}{W_{n} W_{n-2}} \cdot \partial \cdots \partial \cdot \frac{W_{1}^{2}}{W_{2} W_{0}} \cdot \partial \cdot \frac{W_{0}}{W_{1}} \cdot f=\text { const } \neq 0 .
$$


From this identity and the inequalities (4) it immediately follows that

$$
0=\mathbf{V}_{\gamma}(\text { const }) \geqslant \mathbf{V}_{\gamma}(f)-(n-1) K(\gamma)-\sum_{j=0}^{n-1} \mathbf{V}_{\gamma}\left(\frac{W_{j}^{2}}{W_{j-1} W_{j+1}}\right)
$$

where we put for convenience $W_{-1} \equiv 1$, so that $W_{0} / W_{1} \equiv W_{0}^{2} / W_{-1} W_{1}$. This inequality in turn implies an upper bound for the Voorhoeve index of any nontrivial linear combination $f$ by the weighted sum of the Voorhoeve indices of the Wronskians: using (4), one concludes that

$$
\mathbf{V}_{\gamma}(f) \leqslant(n-1) K(\gamma)+4 \sum_{j=1}^{n-2} \mathbf{V}_{\gamma}\left(W_{j}\right)+3 \mathbf{V}_{\gamma}\left(W_{n-1}\right)+\mathbf{V}_{\gamma}\left(W_{n}\right)
$$

We will modify these simple arguments to prove the main result of the paper.

4.2. Proof of Theorem 1. Let $F: U \rightarrow \mathbb{C}^{n}$ be a holomorphic curve with the boundary $\Gamma=F(\gamma), \gamma=\partial U$, and denote by $w_{1}, \ldots, w_{n}$ the weights occurring in (9): $w_{1}=\cdots=w_{n-2}=4, w_{n-1}=3, w_{n}=1$.

1. By Corollary 2, one can choose a complex coordinate system (i.e. a complete flag in $\mathbb{C}^{n}$ ) in such a way that the weighted sum of pseudoinflections of projections of the curve $\Gamma$ on the coordinate subspaces $E_{j}$ will not exceed the weighted sum of the complex Frenet curvatures. Recall that in the chosen coordinate system, the pseudoinflection $\mathbf{I}\left(\Gamma_{k}\right)$ is the variation of argument of the function $\Delta_{k}(t)$, the Wronski determinant of $k$ composite functions $x_{1}(z(t)), \ldots, x_{k}(z(t))$, where $z(t)$ is any fixed parametrization of the boundary $\gamma=\partial U$.

2. Any affine combination of the coordinate functions $\mu_{0}+\mu_{1} x_{1}(z)+\cdots+$ $\mu_{n} x_{n}(z)$ satisfies a linear ordinary differential equation similar to (8) but of order $n+1$ rather than $n$. The Wronskians $W_{k}$ occurring in (8) are to be replaced by the Wronski determinants of the functions $1, x_{1}(z), \ldots, x_{k-1}(z)$, which are obviously equal to the Wronski determinants of the derivatives $x_{1}^{\prime}(z), \ldots, x_{k-1}^{\prime}(z): W_{1}=$ $x_{1}^{\prime}, W_{2}=x_{1}^{\prime} x_{2}^{\prime \prime}-x_{1}^{\prime \prime} x_{2}^{\prime}$ etc. Recall that by convention we put $W_{0} \equiv W_{-1} \equiv 1$.

From $\S 4.1$ it follows that the Voorhoeve index of any affine combination $f$ of the coordinate functions of $F$, and hence $2 \pi$ times the number of isolated intersections between $F$ and any affine complex hyperplane in $\mathbb{C}^{n}$, must satisfy the inequality $\mathbf{V}_{\gamma}(f) \leqslant n K(\gamma)+\sum_{k=0}^{n-1} \mathbf{V}_{\gamma}\left(W_{k}^{2} / W_{k-1} W_{k+1}\right)$.

3. It remains to replace the Wronskians $W_{k}$ in this inequality by the pseudoinflections of the corresponding projections. Using the identities (5) and then (4), for an arbitrary affine combination $f$, we have

$$
\begin{aligned}
2 \pi \boldsymbol{\Omega}(F) \leqslant \sup _{f} \mathbf{V}_{\gamma}(f) & \leqslant n K(\gamma)+\sum_{k=0}^{n-1} \mathbf{V}_{\gamma}\left(\frac{\Delta_{k}^{2}(z(t))}{\Delta_{k-1}(z(t)) \Delta_{k+1}(z(t))} \cdot \dot{z}(t)\right) \\
& \leqslant 2 n K(\gamma)+\sum_{k=1}^{n} w_{k} \mathbf{V}\left(\Delta_{k}\right)=2 n K(\gamma)+\sum_{k=1}^{n} w_{k} \mathbf{I}\left(\Gamma_{k}\right) .
\end{aligned}
$$


The weighted sum of the pseudoinflections in the right hand side is majorized by the weighted sum of integral curvatures by the choice of the coordinate system.

\section{Appendix: improved oscillation bounds for curves in $\mathbb{R}^{n}$}

Using the Rolle-Voorhoeve inequality (4) instead of the usual Rolle lemma allows one to improve the estimates from [1], at least for real analytic curves in $\mathbb{R}^{n}$. This concerns the assertion of Lemma 4 from $[1$, p. 182] and affects all the subsequent estimates. In the notations of $\S 4.1$, assume that all functions $f_{1}, \ldots, f_{n}$ are real analytic on $[0, \ell]$, and denote by $\mathbf{N}(f)$ the number of zeros of a function $f$ on $[0, \ell]$, counted with their multiplicities. Example 2 from $\S 2.2 .2$ and the inequality (9) imply that for any nonzero linear combination $f=\mu_{1} f_{1}+\cdots+\mu_{n} f_{n}$ we have

$$
\mathbf{N}(f) \leqslant(n-1)+4 \mathbf{N}\left(W_{1}\right)+\cdots+4 \mathbf{N}\left(W_{n-2}\right)+3 \mathbf{N}\left(W_{n-1}\right)+\mathbf{N}\left(W_{n}\right) .
$$

For $\ell$-periodic functions the term $n-1$ disappears (see the remark in §2.2.2). This in turn implies the following result substantially improving Theorem 2 from [1]. Recall that for a real curve in $\mathbb{R}^{n}$ the hyperinflection is the number of points where the osculating frame degenerates [1].

Theorem 2. The number of intersections between a real analytic connected curve $\Gamma \subset \mathbb{R}^{n}$ and an arbitrary affine hyperplane, does not exceed the sum $\delta n+\nu(\Gamma)+\frac{1}{\pi}\left[3 K_{n-1}(\Gamma)+4 K_{n-2}(\Gamma)+\cdots+4 K_{1}(\Gamma)\right]$, where $K_{1}, \ldots, K_{n-1}$ are integral Frenet curvatures of the curve, $\nu(\Gamma)$ is the number of hyperinflection points and the index $\delta \in\{0,1\}$ is zero for closed curves and 1 otherwise.

The proof of this result remains exactly the same as in [1] after the above improvement of Lemma 4. Moreover, all subsequent results of [1] concerning rotation of curves around subspaces of codimension $\geqslant 2$, can be improved in a similar way based on Theorem 2 . We leave the details to the reader.

\section{References}

1. D. Novikov and S. Yakovenko, Integral Frenet curvatures and oscillation of spatial curves around affine subspaces of a Euclidean space, J. Dynamical and Control Systems 2 (1996), no. 2, 157-191.

2. E. Calabi, Metric Riemann surfaces, Contributions to the theory of Riemann surfaces, Ann. of Math. Studies, vol. 30, Princeton University Press, Princeton, N. J., 1953, pp. 7785 .

3. A. Khovanskiu and S. Yakovenko, Generalized Rolle theorem in $\mathbb{R}^{n}$ and $\mathbb{C}$, J. Dynamical and Control Systems 2 (1996), no. 1, 103-123.

4. M. Voorhoeve, A generalization of the Descartes' rule, J. London Math. Soc. (2) 20 (1979), $446-456$.

Mathematics Department, Princeton University, Princeton, N.J. 08544, U.S.A.

E-mail address: denadler@math.princeton.edu

Department of Theoretical Mathematics, The Weizmann Institute of Science, REHOVOT 76100, ISRAEL

E-mail address: yakov@wisdom.weizmann.ac.il 